\title{
Editorial
}

\section{La vía política indígena: Agencia y nuevas formas de politización en América Latina}

\author{
Christian Martínez Neira \\ Universidad de Los Lagos, Santiago, Chile. \\ Email: christian.martinez@ulagos.cl \\ Patricia Rodríguez \\ Ithaca College, Nueva York, Estados Unidos \\ Email: prodriguez@ithaca.edu \\ Miguel Leone Jouaunny \\ Universidad de Buenos Aires, Buenos Aires, Argentina. \\ Email: miguelleone@hotmail.com
}

En el último decenio, en Latinoamérica hemos asistido a importantes cambios en materia indígena. Algunos de ellos han sido promovidos por la legislación internacional y, otros, por las propias prácticas y movilizaciones de comunidades y organizaciones indígenas. Sobre todo a partir de la ratificación del Convenio 169 de la OIT, y la Declaración sobre pueblos indígenas de Naciones Unidas, han habido modificaciones en los marcos normativos, las instituciones y en las políticas públicas. Estas han incluido desde distintas formas de reconocimiento constitucional, la aplicación de la consulta previa y, la instalación de legislaciones y políticas sectoriales. Por su parte, las organizaciones indígenas han utilizado un amplio abanico de repertorios, lo que incluye desde su participación en distintas instancias estatales, su incorporación a procesos electorales o diversas formas de autonomía según sea el caso. Las experiencias son muy variadas y, muchas veces han entrado en tensión entre ellas (Lucero, 2006; Madrid, 2012).

El ciclo de los commodities aceleró las actividades extractivistas y sus impactos en las comunidades locales y regionales (Svampa, 2013; Bebbington, 2014; De la Fuente, Ricaldi y Saldomando, 2017). En especial la minería, las actividades petroleras y la producción energética han tenido fuertes consecuencias medioambientales, sociales, culturales y políticas, que han presionado sobre el uso de los bienes naturales desde el agua a las tierras y bosques. Esto ha producido una gran cantidad de conflictos de escala territorial donde participan actores indígenas y no indígenas en contextos con diferentes orientaciones institucionales y capacidad del Estado (Silva, 2016; Martínez y Delamaza, 2018).

Los nuevos contextos estatales, así como la expansión de las actividades extractivistas han generado un nuevo cuadro en el cual situar las luchas clásicas de los pueblos indígenas del continente. De las tradicionales luchas por el acceso a las tierras, la defensa de sus comunidades de la presión de latifundistas y colonos o las dificultades para el acceso a las instituciones públicas, se ha dado paso a nuevos conflictos centrados en la defensa de los territorios de la depredación ambiental, el uso de los bienes comunes, la presión sobre las economías y las culturas locales.

Los casos aquí examinados escapan a los grandes "paradigmas" con que se han abordado las movilizaciones indígenas en el continente: la vía insurreccional chiapaneca o la vía electoral ecuatoriana o 
boliviana. En torno a ambos paradigmas se ha concentrado la mayor parte de la producción y la discusión académica sobre la politización indígena en América Latina (Van Cott, 2005; Yashar, 2005; Bengoa, 2009; González, Burguete y Ortiz, 2010; Rice, 2012; Becker, 2015). En uno se pone el acento en las experiencias de autonomía comunitaria y territorial, en el otro, en la construcción de plataformas y agendas políticas electoralmente viables. Unos cuestionan las vías institucionales de participación pública, los otros, las utilizan para hacer cambios en el Estado y en las políticas públicas. Sin perjuicio de lo anterior, en la mayoría de los casos, ambas vías no siempre se presentan de manera irreductible, lo que no obsta el cuestionamiento de sus alcances y aplicación. Por el contrario, vemos como en distintas experiencias producidas en el continente, las comunidades y organizaciones indígenas intentan de diversa manera generar condiciones favorables para ampliar sus capacidades de decisión y gestión de sus territorios, así como de influenciar las decisiones públicas a nivel local, regional e incluso nacional y trasnacional. De esta manera, la vía política indígena combina una redefinición de la agencia a nivel de sus propios territorios, con diversos niveles de interlocución con actores e instituciones no indígena. Esto vuelve más complejo el panorama de la política indígena y presenta un sinnúmero de desafíos que sobrepasan la escala exclusivamente étnica. Entre estos desafíos se plantean: la redefinición de las formas tradicionales de toma de decisiones y de roles dentro de cada comunidad/pueblo, en especial en temas como la participación de la mujer indígena y la de los jóvenes. La elaboración de alianzas con otros actores no indígenas a nivel social y político. La conformación de instancias de representación política más allá de las propias comunidades. La negociación de espacios institucionales de participación, incluyendo diversas formas de competencia electoral. La capacidad de coordinar los distintos actores del territorio para negociar y/ o hacer frente a los proyectos de intervención en éste. Sólo por nombrar los más relevantes.

Esto ha producido distintas estrategias en los actores indígenas que varían según sus contextos nacionales y regionales. En la mayoría de los casos se combinan alianzas locales y extralocales, con una cierta autonomía local. En todas ellas, han surgido nuevas formas de politización y agencia indígena que articulan distintas escalas, alianzas de actores y relaciones con la institucionalidad pública que producen nuevos horizontes para la investigación. ¿De qué manera estos nuevos contextos producen desafíos para los liderazgos y procesos de toma de decisiones en las comunidades? ¿Qué nuevas estrategias se elaboran para conectar diversos niveles de articulación entre actores? ¿Cuáles son las nuevas orientaciones que se producen con respecto a otros actores no indígenas? ¿Qué vínculos se negocian con las instituciones estatales y no estatales a diferente escala? ¿Hasta qué punto este nuevo contexto redefine el clivaje étnico? ¿Qué nuevos argumentos se elaboran? ¿Qué nuevas formas de politización se producen y qué nuevos repertorios se utilizan? También ¿Cómo se pueden abordar estos temas desde las ciencias sociales y qué desafíos de interdisciplinariedad plantean?

Es bastante evidente que no sólo los contextos han cambiado, sino también, las mismas capacidades y aprendizajes que han logrado las comunidades y organizaciones indígenas para negociar con terceros, construir alianzas, utilizar los espacios institucionales y, en general, posicionarse en la escena pública. En este marco de discusión, este Dossier presta especial atención a la agencia indígena, es decir, a la capacidad de los actores étnicos para actuar sobre sí mismos y su entorno. Según cada contexto local y nacional, los pueblos originarios están haciendo uso de una amplia batería de opciones y repertorios que combinan formas tradicionales con otras emergentes de acción. Esto incluye nuevas formas de hacer uso del derecho consuetudinario hasta la utilización y/o combinación con el derecho estatal; la realización de alianzas con actores no convencionales y revisitar el sistema político a partir de negociaciones pragmáticas; ejercer distintos grados de gestión de sus territorios y discutir temas generales de la sociedad; apelar a marcos interpretativos étnicos y su combinación con nuevas extensiones semánticas que hablan de ambientalismo, patrimonio, derecho internacional, derechos humanos y democracia; entre otros.

Con este 'lente de aproximación’, el presente Dossier profundiza en el conocimiento y en las consecuencias políticas de estas nuevas formas de agencia indígena, ya sea para los propios indígenas, como para la ampliación de los procesos democráticos y de las relaciones entre la sociedad civil y el Estado. Por medio del estudio de casos concretos y su relación con teorías más amplias, los textos que integran este número de la Polis, Revista Latinoamericana analizan diversas formas de politización donde participan los indígenas. La riqueza del mismo se asienta tanto en los aportes originales que los artículos hacen sobre los temas abordados, como sobre la variedad de espacios regionales y nacionales que ellos presentan. Así, los artículos permiten problematizar sobre las nuevas formas que adquiere la agencia indígena en países tan diversos de América 
Latina como México, Chile, Argentina, Ecuador y Venezuela. Los artículos que se presentan a continuación se concentran en cuatro grandes temas: la relación entre las organizaciones indígenas y gobiernos de izquierda que acentuaron una economía extractiva; el co-manejo de territorios por parte de comunidades e instancias estatales; la agencia comunitaria frente a bienes comunes y el medio ambiente; para finalmente abordar, el agenciamiento internacional de organizaciones indígenas y los procesos de auto-identificación.

Dentro del tema de nuevas conexiones y tensiones con lo político, Julimar Mora Silva y Fidel Rodríguez Velásquez muestran, en "la Amazonía en disputa: agencias políticas y organizaciones indígenas de la Amazonía venezolana frente al Arco Minero del Orinoco", que las relaciones Estado-grupos indígenas-aliados tienden a cambiar cuando se produce la crisis del modelo rentista basado en la explotación de recursos naturales. La crisis de este modelo, en vez de frenar o reorientar las políticas estatales a este respecto, abrió espacios para que el gobierno intensificara el extractivismo y la institucionalización de la represión para-estatal. De esta manera, se deterioraron los compromisos territoriales y de derechos humanos previamente comprometidos con las organizaciones indígenas. Así, se generaron nuevas dinámicas entre indígenas y grupos aliados, que resultaron en el quiebre electoral con el gobierno chavista. En este sentido, el artículo entrega insumos para revisar las condiciones en que es posible producir alianzas y acuerdos entre actores indígenas y no indígenas, en contextos extractivos y autoritarios. También, sobre la degradación de la institucionalidad indígena cuando se depende de éstos contextos. En especial, en temas como la soberanía y la consulta previa, por ejemplo.

Una crisis de índole similar sucedió en el caso del Ecuador, como señalan Ivette Vallejo Real y Corinne Duhalde Ruiz, en su artículo "las mujeres indígenas amazónicas: actores emergentes en las relaciones Estado - organizaciones indígenas amazónicas, durante el gobierno de la Alianza País en el Ecuador”. Al igual que en Venezuela, hubo un primer momento en que las organizaciones indígenas lograron acuerdos con el gobierno progresista. Sin embargo, esa alianza se rompe en el año 2013, cuando el gobierno de Rafael Correa decide intensificar la explotación de petróleo en la Amazonía ecuatoriana. Primero cambia la ley minera y de hidrocarburos, luego, licita bloques de explotación que se había acordado no explotar para cuidar el medio ambiente. Las autoras muestran cómo las organizaciones indígenas se dividen ante el intervencionismo de los partidos políticos de gobierno y cómo los impactos de la actividad extractiva afecta de manera diferenciada a hombres de mujeres. Los hombres ven en la industria extractiva una posibilidad laboral, en cambio las mujeres, tienden a mantenerse en sus actividades domésticas a cargo de los cultivos y la alimentación de los hijos. Este impacto diferencial, sumado al contexto de división de las organizaciones indígenas habría generado las condiciones para que las organizaciones de mujeres se hayan fortalecido en torno a la defensa ambiental de la Amazonía. Con todo, el caso ecuatoriano y el venezolano muestran los impactos de la industria extractiva en la institucionalidad y la capacidad de sostener acuerdos políticos entre las organizaciones indígenas y el Estado. Pero también, cómo el cambio en los contextos y la relación entre actores hace emerger nuevas formas de politización y agencia indígena.

Una situación distinta son los casos de experiencias de acuerdos de co-manejo de territorios entre indígenas y la institucionalidad estatal, como así lo muestran Samanta Guiñazú, Florencia Trentini y Nadia Ameghino en "agencia(s) indígena(s) en políticas públicas participativas en Norpatagonia: políticas de comanejo y relevamiento territorial”. En el contexto argentino, ese empoderamiento e ímpetus de participación ocurre a través de proyectos de co-manejo de recursos y territorios dentro de la conservación de parques nacionales y de empoderamiento territorial de comunidades indígenas para frenar los desalojos. Indígenas y actores institucionales accionan proyectos conjuntos, resultando en la profundización de la agencia indígena en la formulación de reconocimiento de derechos y cambios en la relación estado y sociedad civil, así como en el avance hacia una nueva forma de hacer política. Pero también, en el surgimiento de tensiones entre el empoderamiento indígena y la cooptación estatal. En ese sentido, plantean entender estas áreas de co-manejo no como algo dado, sino como un campo en disputa que establece límites pero también, posibilidades de agencia sobre el territorio, los recursos y la ampliación de derechos.

Frecuentemente, las cosmovisiones de los pueblos originarios entienden que el territorio no sólo es el suelo que se pisa sino también el aire, los ríos y las lagunas, el espacio plural y espiritual en que se habita colectiva e históricamente. Entre los mapuche williche, el mar forma parte inescindible de la territorialidad del pueblo. El texto de Daniel Grimaldi, "Etnopolítica del espacio marítimo y el rol de la Identidad Territorial 
Lafkenche en la solicitud de espacios costeros marítimos para pueblos originarios”, retoma esta dimensión de la existencia mapuche y se concentra en analizar cómo funcionó la acción de la Identidad Territorial Lafkenche (ITL) en ese campo de conflictividad. El autor revisita la participación que la ITL tuvo en la elaboración de dispositivos legales que garantizaran los derechos de las comunidades. La Ley de Espacios Costeros Marítimos de Pueblos Originarios (ECMPO), promulgada en 2008, fue el resultado de un intenso proceso de negociaciones entre la ITL y el Estado chileno. Es un punto a destacar que el material que nutre las reflexiones ha sido registrado por el autor a instancias de su propia experiencia de intervención en las políticas indigenistas del Ministerio de Desarrollo de Chile, entre 2014 y 2018. Desde allí, Grimaldi subraya que los lafkenche de la ITL han tenido una gran capacidad para utilizar estratégicamente los "espacios interculturales" abiertos en el ejercicio de la estatalidad chilena. En ese sentido, al igual que el caso de los parques nacionales antes señalado, los espacios de co-manejo afirman un derecho, pero también los delimitan en sus alcances. Con todo, son espacios que interconectan institucionalidades, recursos, derechos y actores, abriendo las posibilidades de acción indígena en nuevos campos y escalas.

El agenciamiento de comunidades indígenas frente a proyectos extractivos es abordado por Deborah Pragier en "comunidades indígenas frente a la explotación de litio en sus territorios: contextos similares, respuestas distintas". La autora intenta responder por qué comunidades que están muy cerca de sí, poseen tradiciones culturales y contextos institucionales muy semejantes tomaron decisiones distintas ante la instalación de empresas extractivas de litio en el norte argentino. En un caso las comunidades aceptaron la instalación extractiva, en otro, lo rechazaron. Sostiene que la respuesta hay que buscarla en la producción de encuadres o framing que permitirían elaborar perspectivas o respuestas ante determinadas situaciones. Señala que se habrían elaborado dos encuadres, uno dependiente y otro autonomista, los que a su vez habrían desencadenado respectivamente demandas de retribución y de reconocimiento. Aquí la pregunta interesante pareciera ser no solo los contenidos de quienes asumen uno u otro encuadre, sino por qué se produce aquello. En el primer caso se señalan argumentos en torno al desarrollo versus empoderamiento local. En el segundo, sobre la antigüedad de la titulación de las tierras. Como sea, en ambos casos se trata de hipótesis argumentales que nos desafían a seguir profundizando en el tema, pero que nos muestran cómo en los procesos de negociación con empresas extractivas se gatillan experiencias previas de las comunidades y sus procesos reivindicativos.

Por su parte, el texto de Edgar Pérez Ríos se titula "De pinos y motosierras: revisión crítica al aprovechamiento forestal comunal en San Jerónimo Coatlán, Oaxaca” y avanza sobre las tonalidades que adquiere la agencia indígena sobre las posibilidades de aprovechamiento de los recursos madereros en el Municipio de San Jerónimo Coatlán, en la Sierra Sur de Oaxaca, México. Apoyándose en un valioso trabajo con fuentes documentales y testimoniales, el autor consigue mostrar cómo, a finales del siglo XX, se crearon comités locales para el aprovechamiento forestal impulsados por la comunidad zapoteca local, disputando el recurso maderero a empresas privadas concesionadas en la región. Los análisis de Pérez Ríos están enriquecidos por su propia pertenencia a estos espacios comunitarios e invitan a revisar cómo pueden ser reconfiguras las relaciones que la comunidad mantiene con el bosque/territorio. El texto muestra que la comunidad ha sabido abandonar parcialmente las actividades de agricultura para dedicarse, en cambio, al aprovechamiento forestal. Con ello, Pérez Ríos aporta elementos interesantes para problematizar aquellas visiones un tanto idílicas que reconocen en lo indígena una pura idea de preservación ecológica del "medioambiente”. También muestra las tensiones que se producen al interior de la comunidad por el uso del bosque, donde una parte de ella lo explota independientemente de las decisiones comunitarias.

Finalmente, Pablo Barnier-Khawam presenta el estudio "la internacionalización de los mapuche: entre pueblo indígena y nación”. El autor muestra cómo ciertas organizaciones indígenas, en este caso mapuche, fueron creando instancias a nivel internacional para incidir tanto en las instituciones internacionales como Naciones Unidas, así como en la propia discusión de la estrategia política mapuche en Chile. El origen de estas organizaciones es el exilio que produjo la dictadura chilena principalmente en Europa. Revisa el fragmentario material que hay a este respecto y lo completa con algunas entrevistas. Si bien en un primer momento se concentra en los asuntos organizacionales, luego profundiza en cómo este proceso podría haber incidido en la discusión sobre la autodefinición política como pueblo indígena o como nación. Lo relevante de esta aproximación es que muestra cómo se produce una cierta capacidad de acción en una escala poco considerada en los estudios indígenas: el activismo internacional indígena. Aunque dicho alcance también desnude debilidades a la 
hora de hablar de representación y construcción organizada de la agenda indígena. Con todo, poco se conoce de este nivel de activismo y sus desafíos organizacionales.

\section{Bibliografía}

Bebbington, A. et al (2014). SubterraneanStruggles: New Dynamics of Mining, Oil, and Gas in LatinAmerica. Austin, US: University of Texas Press.

Becker, M. (2015). ¡Pachakutik! Movimientos indígenas, proyectos políticos y disputas electorales en el Ecuador. Quito, Ecuador: Flacso-AbyaYala.

Bengoa, J. (2009). ¿Una segunda etapa de la emergencia indígena en América Latina? Cuadernos de Antropología Social, (29) 7-22.

De la Fuente, M., Ricaldi, T. y Saldomando, A. (2017). Lógicas de desarrollo, extractivismo y cambio climático. Cochabamba, Bolivia: CESU.

González, M., Burguete, A. y Ortiz, P. (editores.) (2010). La Autonomía a Debate. Quito, Ecuador: Flacso.

Lucero, J. (2006). Representing "Real Indians": TheChallenges of IndigenousAuthenticity and StrategicConstructivism in Ecuador and Bolivia’, LARR, 41 (2).

Madrid, R. (2012). The rice of EthnicPolitics in LatinAmerica. New York, US:Cambridge.

Martínez-Neira, Ch. y Delamaza, G. (2018). Coaliciones interétnicas, framing y estrategias de movilización contra centrales hidroeléctricas en Chile: ¿Qué podemos aprender de los casos de Ralco y Neltume?”, MiddleAtlanticReview of Latin American Studies, 2 (1) 68-96.

Rice, R. (2012). The new politics of protest. Tucson, US: UAP.

Silva, E.(2016). Patagonia, withoutDams! Lessons of a David vs. GoliathCampaign.TheExtractive Industries and Society, 3(4) 947-957.

Svampa, M. (2013). Consenso de los commodities y lenguajesde valoración en América Latina, Nueva Sociedad, (244).

Van Cott, D.L. (2005). From movements to parties in Latin America.Cambridge, US:Cambridge UniversityPress.

Yashar, D. (2005). ContestingCitizenship in LatinAmerica. New York, US: CambridgeUniversityPress. 